\title{
WAVE FRONTS OF ULTRADISTRIBUTIONS VIA FOURIER SERIES COEFFICIENTS
}

\author{
DIJANA DOLIĆANIN-DJEKIĆ, SNJEZANA MAKSIMOVIĆ, AND PETAR SOKOLOSKI
}

\begin{abstract}
We shall use the properties of the product of periodic ultradistributions and give a new description of the wave front of an ultradistribution $f \in \mathscr{D}^{* \prime}\left(\mathbb{R}^{d}\right)$ in terms of Fourier series coefficients.
\end{abstract}

\section{INTRODUCTION}

We have analyzed in [14] the wave front and the Sobolev wave front for distributions through the Fourier coefficients of their appropriate localizations. In this paper we extend the ideas of [14] to ultradistribution spaces. More precisely, we analyze the microlocal properties of an ultradistribution $f$ at $x_{0} \in \mathbb{R}^{d}$ which are determined through the Fourier series expansion of periodizations of $\varphi f$, where $\varphi$ is a cut-off function near $x_{0}$.

Spaces of periodic ultradistributions have been studied mostly in the last 30 years of the last century. We refer here just a few of the papers and books [5, 6, $7,15]$ and also to the papers $[18,17]$ for the discrete wave fronts which provide another approach to the microlocal analysis. We refer to $[3,4,10,11,16]$ for a new approach to the analysis of wave fronts and to $[1,2,12,19,20,22]$ for the theory of periodic distributions. The main difference between the study of periodic distributions and periodic ultradistributions is in the weight functions we use and the consequences. This will be explained in this paper.

1.1. Notation. We follow our notation from [14]. For $x=\left(x_{1}, \ldots, x_{d}\right) \in \mathbb{R}^{d}$, we write $|x|=\left(x_{1}^{2}+\ldots+x_{d}^{2}\right)^{1 / 2}$ and $\langle x\rangle=\left(1+|x|^{2}\right)^{1 / 2}$. Let $0<\eta \leq 1$. We will use the notation

$$
I_{\eta, x}=\prod_{j=1}^{d}\left(x_{j}-\eta / 2, x_{j}+\eta / 2\right) \text { and } I_{\eta}:=I_{\eta, 0} .
$$

We refer to [13] for the spaces of ultradifferentiable functions and their duals, spaces of ultradistributions. In our case, we consider spaces determined by the Gevrey sequence of positive numbers $M_{p}=p !^{s}, p \in \mathbb{N}_{0}$ for some $s>1$. The sequences of this type satisfy the conditions $(M .1),(M .2),(M .3)$ of Komatsu [13].

2010 Mathematics Subject Classification. 35A18; 46F30, 46T30.

Key words and phrases. Wave fronts, Fourier series, multiplication of ultradistributions. 
Recall that functions or ultradistributions on $\mathbb{R}^{d}$ are periodic of period 1 in each variable, if $f(x+n)=f(x)$, for all $x \in \mathbb{R}^{d}, n \in \mathbb{Z}^{d}$. We put $e_{y}$ for $e_{y}(x)=e^{2 \pi i y \cdot x}$, $y \in \mathbb{R}^{d}$. We will consider periodic extensions of localizations of ultradistributions around a point $x_{0} \in \mathbb{R}^{d}$, so if an ultradistribution $g$ is supported by $I_{\eta, x_{0}}$, with $0<\eta<1$, we shall write $g_{p}(x):=\sum_{n \in \mathbb{Z}^{d}} g(x+n)$ for its periodic extension.

1.2. Spaces of ultradifferentiable functions and their duals. The space of periodic test functions $\mathscr{P}^{*}=\mathscr{P}^{*}\left(\mathbb{R}^{d}\right)$ where $*=(s)$, (respectively $*=\{s\}$ ) (Beurling and Roumieu cases, respectively) consists of all the ultradifferentiable periodic functions $\varphi$ of Beurling and Roumieu classes, respectively. It is proved in [7] that $\varphi$ is periodic ultradifferentiable function of order $s$ of Roumieu type $\mathscr{P}\{s\}\left(\mathbb{R}^{d}\right)$, (resp., Beurling type $\mathscr{P}^{(s)}\left(\mathbb{R}^{d}\right)$ ) if and only if

$$
\sum_{n \in \mathbb{Z}^{d}}\left|\varphi_{n}\right|^{2} \exp \left(2 \alpha^{1 / s}|n|^{1 / s}\right)<\infty
$$

for some (resp., any) $\alpha>0$, where $\varphi_{n}=\int_{I_{1}} \varphi(x) e^{-2 \pi i n \cdot x} d x=\left\langle\varphi, e_{-n}\right\rangle, n \in \mathbb{Z}^{d}$. The dual space of $\mathscr{P}^{*}$, the space of periodic ultradistributions, is denoted by $\left(\mathscr{P}^{*}\right)^{\prime}$. Recall [7]: $f=\sum_{n \in \mathbb{Z}^{d}} f_{n} e_{n}$ is a periodic ultradistribution of order $s$ of Roumieu (Beurling) type if and only if $\sum_{n \in \mathbb{Z}_{d}}\left|f_{n}\right|^{2} \exp \left(-2 \alpha^{1 / s}|n|^{1 / s}\right)<\infty$, for any (some) $\alpha>0$. If $f=\sum_{n \in \mathbb{Z}^{d}} f_{n} e_{n} \in\left(\mathscr{P}^{*}\right)^{\prime}$ and $\varphi=\sum_{n \in \mathbb{Z}^{d}} \varphi_{n} e_{n} \in \mathscr{P}^{*}$, then $\langle f, \varphi\rangle=\sum_{n \in \mathbb{Z}^{d}} f_{n} \varphi_{-n}$.

In the sequel, we will consider only Roumieu type basic spaces and their duals. The corresponding results for the Beurling type spaces are similar and even easier to prove. if

Let $\omega, \nu$ be positive functions over $\mathbb{Z}^{d}$. We call the function $\nu$ submultiplicative

$$
\nu(m+n) \leq \nu(m) \nu(n), \forall m, n \in \mathbb{Z}^{d} .
$$

The function $\omega$ is a $\nu$-moderate weight if there is a constant $C$ such that

$$
\omega(m+n) \leq C \omega(m) \nu(n), \forall m, n \in \mathbb{Z}^{d} .
$$

For a fixed submultiplicative function $\nu$, the set of all $\nu$-moderate weights is $\mathscr{M}_{\nu}$. Following [11], for $s>1$,

$$
\mathscr{M}_{\{s\}}\left(\mathbb{Z}^{d}\right)=\left\{\omega \in \mathscr{M}_{\nu}:(\exists C>0)(\exists k>0)\left(\forall n \in \mathbb{Z}^{d}\right)\left(\nu(n) \leq C e^{k|n|^{1 / s}}\right)\right\} .
$$

For $\omega \in \mathscr{M}_{\{s\}}\left(\mathbb{Z}^{d}\right)$, we define

$$
\mathscr{P} l_{\omega}^{q}=\left\{f \in\left(\mathscr{P}^{\{s\}}\right)^{\prime}:\left\{f_{n} \omega(n)\right\}_{n \in \mathbb{Z}^{d}} \in l^{q}, \text { where } f_{n}=\left\langle f, e_{-n}\right\rangle\right\}
$$

supplied by the norm $\|f\|_{\mathscr{P} l_{\omega}^{q}}=\left\|\left\{f_{n} \omega(n)\right\}\right\|_{l^{q}}$. We consider from now on only values $q \geq 1$. Clearly, $\mathscr{P} l_{\omega_{1}}^{q_{1}} \subseteq \mathscr{P} l_{\omega_{2}}^{q_{2}}$, if $q_{1} \leq q_{2}$ and $\omega_{2} \leq C \omega_{1}$. We will also consider the local space $\mathscr{P} l_{\omega, l o c}^{q}$ consisting of distributions $f \in\left(\mathscr{D}^{\{s\}}\right)^{\prime}\left(\mathbb{R}^{d}\right)$ such that the periodic extensions $(\varphi f)_{p} \in \mathscr{P} l_{\omega}^{q}$, for all $x_{0} \in \mathbb{R}^{d}$ and $\varphi \in \mathscr{D}^{\{s\}}\left(I_{1, x_{0}}\right)$. Its topology is defined via the family of seminorms $\|f\|_{x_{0}, \varphi}=\left\|(\varphi f)_{p}\right\|_{\mathscr{P} l_{\omega}^{q}}$, where $x_{0} \in \mathbb{R}^{d}$ and $\varphi \in \mathscr{D}^{\{s\}}\left(I_{1, x_{0}}\right)$. For the sake of completeness, we give the following elementary proposition (its proof also shows that the definition of $\mathscr{P} l_{\omega, l o c}^{q}$ is consistent). 
Proposition 1.1. $\mathscr{P} l_{\omega}^{q} \subset \mathscr{P} l_{\omega, l o c}^{q}$.

Proof. Let $f \in \mathscr{P} l_{\omega}^{q}$ and $\varphi \in \mathscr{D}^{\{s\}}\left(I_{1, x_{0}}\right)$, then $(\varphi f)_{p}=\varphi_{p} f$. Write $f=\sum_{n} f_{n} e_{n}$ and $\varphi_{p}=\sum_{n} \varphi_{n} e_{n} \in \mathscr{P}^{\{s\}}$. By (1.2) and the generalized Minkowski inequality, we have

$$
\begin{aligned}
\left\|\varphi_{p} f\right\|_{\mathscr{P} l_{\omega}^{q}} & \leq C\left(\sum_{n}\left(\sum_{j}\left|\varphi_{j}\right| \nu(j)\left|f_{n-j}\right| \omega(n-j)\right)^{q}\right)^{1 / q} \\
& \leq C\left\|\varphi_{p}\right\|_{\mathscr{P} l_{\nu}^{1}}\|f\|_{\mathscr{P} l_{\omega}^{q}<\infty}
\end{aligned}
$$

For fixed $s>1$, set $\omega_{k}(n)=e^{k|n|^{1 / s}}, k \in \mathbb{R}$. For convenience, we write $\mathscr{P} l_{k}^{q}:=$ $\mathscr{P} l_{\omega_{k}}^{q}$ and $\mathscr{P} l_{k, l o c}^{q}:=\mathscr{P} l_{\omega_{k}, l o c}^{q}$. We clearly have

$$
\mathscr{P}\{s\}=\bigcap_{k \geq 0} \mathscr{P} l_{k}^{q}=\bigcap_{\omega \in \mathscr{M}_{\{s\}}\left(\mathbb{Z}^{d}\right)} \mathscr{P} l_{\omega}^{q}
$$

and

Moreover,

$$
(\mathscr{P}\{s\})^{\prime}=\bigcup_{k \leq 0} \mathscr{P} l_{k}^{q}=\bigcup_{\omega \in \mathscr{M}_{\{s\}}\left(\mathbb{Z}^{d}\right)} \mathscr{P} l_{\omega}^{q} .
$$

$$
\mathscr{E}\{s\}=\bigcap_{k \geq 0} \mathscr{P} l_{k, l o c}^{q}=\bigcap_{\omega \in \mathscr{M}_{\{s\}}\left(\mathbb{Z}^{d}\right)} \mathscr{P} l_{\omega, l o c}^{q}
$$

and

$$
\left(\mathscr{D}^{\{s\}}\right)_{F}^{\prime}=\bigcup_{k \leq 0} \mathscr{P} l_{k, l o c}^{q}=\bigcup_{\omega \in \mathscr{M}_{\{s\}}\left(\mathbb{Z}^{d}\right)} \mathscr{P} l_{\omega, l o c}^{q},
$$

where $\mathscr{E}^{\{s\}}$ is the space of all ultradifferentiable functions of order $s$ of Roumieu type and $\left(\mathscr{D}^{\{s\}}\right)_{F}^{\prime}$ is the space of ultradistributions of finite order of Roumieu type on $\mathbb{R}^{d}$, that is $g \in\left(\mathscr{D}^{\{s\}}\right)_{F}^{\prime}$ if for some ultradifferential operator $P$ of class $\{s\}$ and some $G$, continuous on $\mathbb{R}^{d}$, there holds $g=P(D) G,[13]$.

1.3. Multiplication within periodic test spaces. We fix two weight functions $\omega \in \mathscr{M}_{\nu}, \nu \in \mathscr{M}_{\{s\}}\left(\mathbb{Z}^{d}\right)$ (cf. (1.2)). Let $f_{1}=\sum_{n \in \mathbb{Z}^{d}} f_{1, n} e_{n} \in \mathscr{P} l_{\omega}^{q_{1}}$ and $f_{2}=$ $\sum_{n \in \mathbb{Z}^{d}} f_{2, n} e_{n} \in \mathscr{P} l_{\nu}^{q_{2}}$. Then we define their product as $f:=f_{1} f_{2}:=\sum_{n \in \mathbb{Z}^{d}} f_{n} e_{n}$, where

$$
f_{n}=\sum_{j \in \mathbb{Z}^{d}} f_{1, n-j} f_{2, j}, \quad n \in \mathbb{Z}^{d} .
$$

This definition allows us to introduce multiplication in the local versions of these spaces. Let $f_{1} \in \mathscr{P} l_{\omega, l o c}^{q_{1}}$ and $f_{2} \in \mathscr{P} l_{\nu, l o c}^{q_{2}}$. Then the product $f:=f_{1} f_{2}$ is defined as follows. Let $x_{0} \in \mathbb{R}^{d}, 0<\eta<1$ and let $\phi \in \mathscr{D}^{\{s\}}\left(I_{1, x_{0}}\right)$ be such that $\phi(x)=1$ for $x \in I_{\eta, x_{0}}$. We define $f_{I_{\eta, x_{0}}} \in\left(\mathscr{D}^{\{s\}}\right)^{\prime}\left(I_{\eta, x_{0}}\right)$ as the restriction of $\left(\phi f_{1}\right)_{p}\left(\phi f_{2}\right)_{p}$ to $I_{\eta, x_{0}}$. Note that different choices of $\phi$ lead to different Fourier coefficients but, by Proposition 1.1, we have $f_{I_{\eta, x_{0}}}=f_{I_{\eta^{\prime}, x_{0}^{\prime}}}$ on $I_{\eta, x_{0}} \cap I_{\eta^{\prime}, x_{0}^{\prime}}$. Thus, by $\left\{f_{I_{\eta, x_{0}}}\right\}$ we have a distribution $f \in \mathscr{P} l_{\omega, l o c}^{q}$ as the product of $f_{1} f_{2}:=f$. 
Proposition 1.2. Let $q, q_{1}, q_{2} \in[1, \infty]$ are such that $\frac{1}{q_{1}}+\frac{1}{q_{2}}=\frac{1}{q}+1$. Then the mappings

$$
\mathscr{P} l_{\omega}^{q_{1}} \times \mathscr{P} l_{\nu}^{q_{2}} \ni\left(f_{1}, f_{2}\right) \mapsto f_{1} f_{2} \in \mathscr{P} l_{\omega}^{q}
$$

and

are continuous.

$$
\mathscr{P} l_{\omega, l o c}^{q_{1}} \times \mathscr{P} l_{\nu, l o c}^{q_{2}} \ni\left(f_{1}, f_{2}\right) \mapsto f_{1} f_{2} \in \mathscr{P} l_{\omega, l o c}^{q}
$$

Proof. The continuity of (1.4) follows from (1.3), while (1.3), Young's inequality and (1.2) imply

$$
\left\|f_{1} f_{2}\right\|_{\mathscr{P} l_{\omega}^{q}} \leq C\left\|f_{1}\right\|_{\mathscr{P} l_{\omega}^{q_{1}}}\left\|f_{2}\right\|_{\mathscr{P} l_{\nu}^{q_{2}}} .
$$

As a corollary, we have:

Corollary 0.1. Let $k, k_{1}, k_{2} \in \mathbb{R}$,

$$
k_{1}+k_{2} \geq 0, \quad k \leq \min \left\{k_{1}, k_{2}\right\} .
$$

Then, the mappings $\mathscr{P} l_{k_{1}}^{q_{1}} \times \mathscr{P} l_{k_{2}}^{q_{2}} \ni\left(f_{1}, f_{2}\right) \mapsto f_{1} f_{2} \in \mathscr{P} l_{k}^{q}$ and $\mathscr{P} l_{k_{1}, l o c}^{q_{1}} \times$ $\mathscr{P} l_{k_{2}, l o c}^{q_{2}} \ni\left(f_{1}, f_{2}\right) \mapsto f_{1} f_{2} \in \mathscr{P} l_{k, l o c}^{q}$ are continuous.

Proof. Assume $k_{1} \geq 0$ and $k=k_{2}$ (it is not a restriction). Clearly $k_{1} \geq\left|k_{2}\right|$ has to hold in order to have $k_{1}+k_{2} \geq 0$. The continuity then follows from Proposition 1.2 with $\omega(n)=e^{k_{2}|n|^{1 / s}}$ and $\nu(n)=e^{k_{1}|n|^{1 / s}}$ because (1.2) holds in this case.

\section{WAVE Front}

As in the case of distributions, our aim is to describe the wave front of an $f \in\left(\mathscr{D}^{\{s\}}\right)^{\prime}\left(\mathbb{R}^{d}\right)$ via the Fourier series coefficients of the periodic extension of an appropriate localization of $f$ around $x_{0} \in \mathbb{R}^{d}$. Recall that $\left(x_{0}, \xi_{0}\right) \notin W F(f)$ if there exist $\psi \in\left(\mathscr{D}^{\{s\}}\right)^{\prime}\left(\mathbb{R}^{d}\right)$ with $\psi \equiv 1$ in a neighborhood of $x_{0}$ and an open cone $\Gamma \subset \mathbb{R}^{d} \backslash\{0\}$ containing $\xi_{0}$ such that

$$
\left.(\exists N>0)\left(\exists C_{N}>0\right)\right)(\forall \xi \in \Gamma)|\widehat{\psi f}(\xi)| \leq C_{N} e^{-N|\xi|^{1 / s}} .
$$

Theorem 1. Let $f \in\left(\mathscr{D}^{\{s\}}\right)^{\prime}\left(\mathbb{R}^{d}\right)$ and $\left(x_{0}, \xi_{0}\right) \in \mathbb{R}^{d} \times\left(\mathbb{R}^{d} \backslash\{0\}\right)$. The following conditions are equivalent:

(i) There exist $\phi \in \mathscr{D}^{\{s\}}\left(I_{\varepsilon, x_{0}}\right)$, with $\varepsilon \in(0,1)$ and $\phi \equiv 1$ in a neighborhood of $x_{0}$, and an open cone $\Gamma$ containing $\xi_{0}$ such that

$$
(\exists N \in \mathbb{N})\left(\exists C_{N}>0\right)\left(\forall n \in \Gamma \cap \mathbb{Z}^{d}\right)|\widehat{\psi f}(n)| \leq C_{N} e^{-N|n|^{1 / s}} .
$$

(ii) $\left(x_{0}, \xi_{0}\right) \notin W F(f)$.

Proof. The proof is similar to the one given in [14] for distributions, but for the sake of completeness we give all the details. By shrinking the conic neighborhood of $\xi_{0}$, we can choose $\psi$ in $(2.1)$ with arbitrarily small support around $x_{0}$. With this $(i i)$ implies $(i)$. So, now it is enough to show that $(i)$ implies $(i i)$. Assume $(i)$. We will prove that there are $\varepsilon^{\prime}$ and an open cone $\xi_{0} \in \Gamma_{1}$ such that

$\left(\forall B\right.$ bounded set in $\left.\mathscr{D}^{\{s\}}\left(I_{\varepsilon^{\prime}, x_{0}}\right)\right)(\forall N>0)\left(\exists C_{N}^{\prime}>0\right)$ 


$$
\left(\forall n \in \Gamma_{1} \cap \mathbb{Z}^{d}\right) \quad \sup _{\varphi \in B}|\widehat{\varphi f}(n)| \leq C_{N}^{\prime} e^{-N|n|^{1 / s}} .
$$

Let $\varepsilon^{\prime}$ be such that $\phi(x)=1$ for every $x \in I_{\varepsilon^{\prime}, x_{0}}$. Let $\Gamma_{1}$ be an open cone with $\xi_{0} \in \Gamma_{1}$ and $\bar{\Gamma}_{1} \subset \Gamma \cup\{0\}$. Let $0<c<1$ be a constant smaller than the distance between $\partial \Gamma$ and the intersection of $\bar{\Gamma}_{1}$ with the unit sphere. Then $\left\{y \in \mathbb{R}^{d}:\left(\exists \xi \in \Gamma_{1}\right)(|\xi-y| \leq c|\xi|)\right\} \subset \Gamma$. Let $B \subset \mathscr{D}^{\{s\}}\left(I_{\varepsilon^{\prime}, x_{0}}\right)$ be a bounded set. Since $\phi \varphi=\varphi, \forall \varphi \in B$ we have that $\widehat{f \varphi}(n)$ are the Fourier coefficients of the periodic distribution $(\phi f)_{p}(\varphi)_{p}$. Thus, for $\varphi \in B$ and $n \in \Gamma_{1} \cap \mathbb{Z}^{d}$,

$$
\begin{aligned}
|\widehat{\varphi f}(n)|=\left|\sum_{j \in \mathbb{Z}^{d}} \widehat{\phi f}(n-j) \widehat{\varphi}(j)\right| & \leq\left(\sum_{|j| \leq c|n|}+\sum_{|j|>c|n|}\right)|\widehat{\phi f}(n-j) \widehat{\varphi}(j)| \\
& =: I_{1}(n)+I_{2}(n)
\end{aligned}
$$

Now we estimate $I_{1}(n)$ :

$$
I_{1}(n)=\sum_{|n-j| \leq c|n|}|\widehat{\phi f}(j)||\widehat{\varphi}(n-j)| \leq C \sup _{|n-j| \leq c|n|}|\widehat{\phi f}(j)|,
$$

where $C$ only depends on $B$. Since $|n-j| \leq c|n|$ implies $|j| \geq(1-c)|n|$, we have

$$
\begin{aligned}
& \sup _{\varphi \in B, n \in \Gamma_{1}} e^{-N|n|^{1 / s}} I_{1}(n) \leq C \sup _{n \in \Gamma_{1}} e^{-N|n|^{1 / s}} \sup _{|n-j| \leq c|n|}|\widehat{\phi f}(j)| \\
& \leq C \sup _{j \in \Gamma_{\xi_{0}}}(1-c)^{-N} e^{-N|j|^{1 / s}}|\widehat{\phi f}(j)|=C(1-c)^{-N} C_{N} .
\end{aligned}
$$

Next we estimate $I_{2}$. For this we use that $|n-j| \leq\left(1+c^{-1}\right)|j|$ if $|j| \geq c|n|$. By the Paley-Wiener theorem, there are $M>0, D>0$ such that

$$
|\widehat{\phi f}(n-j)| \leq D e^{-M|n-j|^{1 / s}}, \quad n, j \in \mathbb{Z}^{d} .
$$

The boundedness of $B \subset \mathscr{D}^{\{s\}}\left(\mathbb{R}^{d}\right)$, implies

$$
\sup _{\varphi \in B} \sum_{j \in \mathbb{Z}^{d}} e^{(M+N)|j|^{1 / s}}|\widehat{\varphi}(j)|=: K_{N}<\infty .
$$

Moreover, we have

$$
\begin{array}{r}
\sup _{n \in \Gamma_{1}} e^{-N|n|^{1 / s}} I_{2}(n) \leq D \sup _{n \in \Gamma_{1}} e^{-N|n|^{1 / s}} \sum_{|j| \geq c|n|} e^{M|n-j|^{1 / s}}|\widehat{\varphi}(j)| \\
\leq D C^{-N}\left(1+c^{-1}\right)^{M} K_{N}, \quad \forall \varphi \in B .
\end{array}
$$

By (2.4) and (2.5), we obtain (2.3).

Now we show that $\left(x_{0}, \xi_{0}\right) \notin W F(f)$ by $(2.3)$. Let $\psi \in \mathscr{D}^{\{s\}}\left(I_{\varepsilon^{\prime}, x_{0}}\right)$ be equal to 1 in a neighborhood of $x_{0}$. Then, the set $B=\left\{\varphi_{t}:=\psi e_{-t}: t \in[0,1)^{d}\right\}$ is a bounded subset of $\mathscr{D}^{\{s\}}\left(I_{\varepsilon^{\prime}, x_{0}}\right)$. This implies

$$
\begin{gathered}
\sup _{t \in[0,1)^{d}}|\widehat{\psi f}(n+t)|=\sup _{t \in[0,1)^{d}} \widehat{\mid \varphi_{t} f}(n) \mid \leq \frac{C_{N}^{\prime}}{e^{N|n|^{1 / s}}}, \quad \forall n \in \Gamma_{1} \cap \mathbb{Z}^{d}, \\
\sup _{\xi \in\left(\Gamma_{1} \cap \mathbb{Z}^{d}\right)+[0,1)^{d}}\left|e^{N|\xi|^{1 / s}} \widehat{\psi f}(\xi)\right| \leq(1+4 d)^{N / 2} C_{N}^{\prime} .
\end{gathered}
$$


Let $\Gamma_{2}$ be an open conic neighborhood of $\xi_{0}$ such that $\bar{\Gamma}_{2} \subset \Gamma_{1} \cup\{0\}$ and let $c^{\prime}$ be such that $\left\{y \in \mathbb{R}^{d}:\left(\exists \xi \in \Gamma_{2}\right)\left(|\xi-y| \leq c^{\prime}|\xi|\right)\right\} \subset \Gamma_{1}$. Then $\Gamma_{2} \cap\left\{\xi \in \mathbb{R}^{d}:|\xi| c^{\prime} \geq\right.$ 1\} $\subset\left(\Gamma_{1} \cap \mathbb{Z}^{d}\right)+[0,1)^{d}$. Thus

$$
\sup _{\xi \in \Gamma_{2}} e^{N|\xi|^{1 / s}}|\widehat{\psi f}(\xi)| \leq \max \left\{C_{N}^{\prime \prime},(1+4 d)^{N / 2} C_{N}^{\prime}\right\}=C_{N}<\infty
$$

where $C_{N}^{\prime \prime}=\sup _{\xi \in \Gamma_{2},|\xi|<1 / c^{\prime}} e^{N|\xi|^{1 / s}}|\widehat{\psi f}(\xi)|$. This shows that $\left(x_{0}, \xi_{0}\right) \notin W F(f)$. The assertion is proved.

\section{REFERENCES}

[1] P. Antosik, J. Mikusiński, R. Sikorski, Theory of distributions. The sequential approach, Elsevier Scientific Publishing Co., Amsterdam; PWN-Polish Scientific Publishers, Warsaw, 1973.

[2] R. Beals, Advanced mathematical analysis. Periodic functions and distributions, complex analysis, Laplace transform and applications. Graduate Texts in Mathematics, No. 12, Springer-Verlag, New York-Heidelberg, 1973.

[3] S. Coriasco, K. Johanson, J. Toft. Local wave-front sets of Banach and Fréchet types, and pseudo-differential operators, Monatsh. Math. 169 (2013), 285-316.

[4] S. Coriasco, K. Johanson, J. Toft. Global wave-front sets of Banach, Fréchet and modulation space types, and pseudo-differential operators, J. Differential Equations 254 (2013), 32283258.

[5] V. I. Gorbačuk, M. L. Gorbačuk, Trigonometric series and generalized functions, Dokl. Akad. Nauk SSSR (4) 257 (1981), 799-804, (Russian).

[6] V. I. Gorbačuk, On Fourier series of periodic ultradistributions,Ukranian Math. J. (2) 34 (1982), 144-150. (Russian).

[7] V. I. Gorbačuk, M. L. Gorbačuk, Boundary value problems for operator differential equations, (Mathematics and its Applications) -Kluwer, 1991.

[8] L. Hörmander, The analysis of linear partial differential operators I: Distribution theory and Fourier analysis, Springer-Verlag, 1983.

[9] L. Hörmander, Lectures on nonlinear hyperbolic differential equations, Springer-Verlag, 1997.

[10] K. Johansson, S. Pilipović, N. Teofanov, J. Toft, Gabor pairs, and a discrete approach to wave-front sets, Monatsh. Math. 166 (2012), 181-199.

[11] K. Johansson, S. Pilipović, N. Teofanov, J. Toft, Micro-local analysis in some spaces of ultradistributions, Publ. Inst. Math. (Beograd) (N.S.) 92(106) (2012), 1-24.

[12] R. P. Kanwal, Generalized functions. Theory and applications, Birkhäuser Boston, Inc., Boston, MA, 2004

[13] H. Komatsu, Ultradistributions. I. Structure theorems and a characterization., J. Fac. Sci. Univ. Tokyo Sect. IA Math. 20 (1973), 25-105.

[14] S. Maksimovic, S. Pilipovic, P.Sokoloski, J. Vindas Wave fronts of distributions via Fourier coefficients.

[15] S. Pilipovic,Structural theorems for periodic ultradistributions, Proc. Amer. Math. Soc. 98 (1986), 261-266.

[16] S. Pilipović, N. Teofanov, J. Toft, Micro-local analysis with Fourier-Lebesgue spaces. Part I, J. Fourier Anal. Appl. 17 (2011), 374-407.

[17] M. Ruzhansky, V. Turunen, Quantization of pseudo-differential operators on the torus, J. Fourier Anal. Appl. 16 (2010), 943-982. -

[18] M. Ruzhansky, V. Turunen, Pseudo-differential operators and symmetries. Background analysis and advanced Topics, Birkhäuser Verlag, Basel, 2010.

[19] L. Schwartz, Théorie des distributions, Hermann, Paris, 1966.

[20] K. Skórnik, Hereditarily periodic distributions, Studia Math. 43 (1972), 245-272. 
[21] J. Vindas, R. Estrada, Distributional point values and convergence of Fourier series and integrals, J. Fourier Anal. Appl. 13 (2007), 551-576.

[22] G. Walter, Pointwise convergence of distribution expansions, Studia Math. 26 (1966), 143154.

Faculty of Technical Sciences in Kosovska Mitrovica, and, State University of Novi Pazar, Novi Pazar, Serbia

Email address: dolicanin_d@yahoo.com

Faculty of Electrical Engineering, University of Banja Luka, Patre 5, 78000 Banja Luka, Bosnia and Herzegovina

Email address: snjezana.maks imovic@etfbl.net

Faculty of Natural Sciences and Mathematics, Ss. Cyril and Methodius University,

Arhimedova 3, 1000 Skopje, Republic of Macedonia

Email address: petar@pmf.ukim.mk 